\title{
Expression of Notch 2 and $A B C C 8$ genes in patients with type 2 diabetes mellitus and their association with diabetic kidney disease
}

\section{ABSTRACT}

Background. The incidence of type 2 diabetes mellitus (T2DM) has increased over the past years and early identification and management of its complications especially diabetic kidney disease (DKD) is of great importance. T2DM and DKD are of multifactorial etiology with contribution of genetic and environmental factors. We aimed to study the expression of ABCC8 and Notch 2 genes in patients with T2DM and to find their association with DKD.

Methods. The present work was carried on 80 patients with T2DM (40 with DKD and 40 without DKD) and 40 healthy subjects as a control group. Real time polymerase chain reaction was used to assess gene expression.

Results. Altered expression of ABCC8 and Notch 2 genes were found in patients with T2DM compared to control group. $A B C C 8$ expression had significant positive correlation with $\mathrm{HbA}_{1 \mathrm{c}}$ while Notch 2 expression had significant positive correlation with fasting plasma glucose and $\mathrm{HbA}_{1 \mathrm{c}}$. Notch 2 expression was significantly higher in patients with DKD compared to those without DKD. Multivariate regression analysis showed that Notch 2 expression had independent relation with increased urinary albumin excretion and

Address for correspondence:

Amr Y. El-Feky

Ramlh station, Alexandria, Egypt

Phone: 00201091532336

e-mail: ammmmmr28@yahoo.com

Clinical Diabetology 2020, 9; 5: 306-312

DOI: $10.5603 /$ DK.2020.0037

Received: 03.05.2020

Accepted: 12.06 .2020 reduced estimated glomerular filtration rate. $A B C C 8$ gene expression did not show significant difference between diabetic patients with DKD compared to those without DKD.

Conclusion. Increased expression of ABCC8 and Notch 2 genes may play a role in pathogenesis of T2DM. Overexpression of Notch 2 gene may have a role in the development of albuminuria and DKD in patients with T2DM which may represent a possible diagnostic tool and a possible therapeutic target. (Clin Diabetol 2020; 9; 5: 306-312)

Key words: type 2 diabetes, $A B C C 8$, Notch 2, diabetic kidney disease, gene expression

\section{Introduction}

Diabetes mellitus (DM) is a major health problem worldwide [1]. It is estimated that more than 400 million adults had DM in 2019 which is predicted to reach 700 million by 2045 [2]. Egypt is considered among the world top 10 countries in the world regarding the number of patients with DM [2].

Type 2 diabetes mellitus (T2DM) accounts for about 90-95\% of all cases of diabetes [3]. T2DM is considered a heterogeneous disease caused by interaction between genetics and environmental factors [4]. The damage precipitated by hyperglycemia on the human vascular tree is the major source of morbidity and mortality in T2DM due to functional and structural organ damage as a result of changes in the vascular system [5].

Diabetic kidney disease (DKD) is a leading cause of end stage renal disease (ESRD) worldwide and more 
than $80 \%$ of cases of ESRD globally are caused by diabetes or hypertension, or a combination of both [2]. DKD may be present at diagnosis of T2DM and the presence of DKD markedly increases cardiovascular risk in patients with DM $[6,7]$.

Diagnosis of DKD is usually made based on the presence of albuminuria and/or reduced eGFR in the absence of symptoms or signs of other primary causes of kidney damage [6]. In light of the high prevalence of T2DM and its deleterious effect on the kidney, early detection and management of DKD are of great clinical importance.

ATP binding cassette, subfamily $C$, member 8 (ABCC8) gene provides instructions for making the sulfonylurea receptor 1 (SUR1) protein [8]. The SUR1 protein is a subunit of the ATP-sensitive potassium channel that controls the secretion of insulin out of beta cells of the pancreas into the bloodstream [9].

Notch 2 gene provides instructions for making Notch 2 protein which is a member of the Notch family of receptors [10]. Notch 2 receptor is expressed in multiple cells and tissues including the pancreas, the liver and the kidney [11].

Studies have discovered multiple variants of genes associated with T2DM but the influence of most of these genes on the disease and on DKD is unclear [12]. Discoveries concerning the genetics of T2DM and DKD may have clinical implications by using these genes as diagnostic or therapeutic targets [13].

In the highlight of the role of genetic factors in the pathogenesis of T2DM and DKD, we aimed in this study to investigate the expression of $A B C C 8$ and Notch 2 genes in Egyptian patients with T2DM and to investigate their association with DKD in these patients.

\section{Methods}

This study was conducted on 80 patients with T2DM who fulfilled the diagnostic criteria of American Diabetes Association (ADA) of T2DM [3]. The patients were divided into two groups, the first group included 40 patients with DKD who fulfilled the criteria of ADA for diagnosis of DKD (based on the presence of albuminuria and/or reduced eGFR with no evidence of primary causes of renal damage) [6] and the second group included 40 patients without DKD. The cases were recruited from the outpatient clinic of diabetes in Alexandria Main University Hospital.

Patients with type 1 diabetes, gestational diabetes, hepatic or cardiac disease, hematological disorders and malignancies, history of hemodialysis or renal transplantation, infection, other acute or chronic autoimmune or metabolic diseases and patients using nephrotoxic drugs or corticosteroids were excluded from this study.
The study also included another 40 healthy subjects who volunteered to join this study as a control group and informed consent was obtained from all the individuals in this study.

To all the studied subjects full history was taken with special emphasis on the duration of diabetes, drug history with emphasis on antidiabetic drugs, antihypertensive drugs, corticosteroids in addition to any nephrotoxic drugs and presence of any chronic diseases especially any renal disorder.

Complete physical examination was done including: heart rate, blood pressure measurement, complete cardiac examination, complete chest examination, measuring anthropometric parameters including body mass index (BMI) which was calculated as weight/ /height ${ }^{2}$ in meter and waist circumference (WC) which was measured at midpoint between the highest point of the iliac crest and lowest point of costal margin at the end of normal expiration.

\section{Laboratory investigations}

Early morning urine sample was obtained for complete urine analysis and assessment of urinary albumin to creatinine ratio.

Blood sampling: sampling was done in the morning (8.00-10.00 am) after an overnight fast of 10 hours. 9 milliliters of venous blood were withdrawn from each subject and divided into the vacutainers for assessment of the following: fasting plasma glucose (FPG), lipid profile (triglycerides (TG), total cholesterol (TC), LDL- cholesterol and HDL-cholesterol, blood urea, serum creatinine, CRP, AST and ALT activity which were analysed using a biochemical analyser (Dimension, Siemens, Germany) using reagent kits supplied by the manufacturer of the analyzer in addition to $\mathrm{HbA}_{1 \mathrm{c}}$ percentage [which was performed using an automated high performance liquid chromatography (HPLC; Tosoh)] and molecular studies.

Molecular studies: included estimation of $A B C C 8$ gene expression via determination of $A B C C 8$ mRNA level in peripheral blood mononuclear cells (PBMC) by real time PCR. Estimation of Notch 2 gene expression via determination of Notch 2 mRNA level in PBMC by real time PCR. Determination of glyceraldehyde3-phosphate dehydrogenase (GAPDH) mRNA level as a housekeeping gene in PBMC by real time PCR.

\section{Calculations}

Calculation of estimated GFR (eGFR) using CKD-EPI equation [14].

Relative expression of target genes ( $A B C C 8$ and Notch 2) was calculated using the comparative CT method ( $2^{-\Delta \Delta} \mathrm{CT}$ method). The method describes the change in expression of the target gene relative to 
Table 1. Demographic data of studied population

\begin{tabular}{|c|c|c|c|c|c|c|c|c|c|c|}
\hline & \multicolumn{6}{|c|}{ Cases with T2DM } & \multicolumn{2}{|c|}{ Control $(n=40)$} & \multirow[t]{3}{*}{$P_{1}$} & \multirow[t]{3}{*}{$\mathbf{P}_{2}$} \\
\hline & \multicolumn{2}{|c|}{$\begin{array}{c}\text { Total } \\
(n=80)\end{array}$} & \multicolumn{2}{|c|}{$\begin{array}{l}\text { With DKD } \\
(n=40)\end{array}$} & \multicolumn{2}{|c|}{$\begin{array}{l}\text { Without DKD } \\
\qquad(n=40)\end{array}$} & \multirow[t]{2}{*}{ No. } & \multirow[t]{2}{*}{$\%$} & & \\
\hline & No. & $\%$ & No. & $\%$ & No. & $\%$ & & & & \\
\hline \multicolumn{11}{|l|}{ Sex } \\
\hline Male & 44 & 55.0 & 21 & 52.5 & 23 & 57.5 & 19 & 47.5 & 0.438 & 0.653 \\
\hline Female & 36 & 45.0 & 19 & 47.5 & 17 & 42.5 & 21 & 52.5 & & \\
\hline \multicolumn{11}{|l|}{ Age (years) } \\
\hline Mean $\pm S D$ & \multicolumn{2}{|c|}{$53.93 \pm 6.63$} & \multicolumn{2}{|c|}{$54.24 \pm 10.18$} & \multicolumn{2}{|c|}{$54.08 \pm 8.54$} & \multicolumn{2}{|c|}{$51.37 \pm 9.29$} & 0.127 & 0.986 \\
\hline \multicolumn{11}{|l|}{ BMI $\left[\mathrm{kg} / \mathrm{m}^{2}\right]$} \\
\hline Mean \pm SD & \multicolumn{2}{|c|}{$31.16 \pm 3.57$} & \multicolumn{2}{|c|}{$31.34 \pm 4.32$} & \multicolumn{2}{|c|}{$30.98 \pm 2.67$} & \multicolumn{2}{|c|}{$27.37 \pm 3.03$} & 0.001 & 0.657 \\
\hline \multicolumn{11}{|l|}{$W C[\mathrm{~cm}]$} \\
\hline Mean $\pm S D$ & \multicolumn{2}{|c|}{$116.0 \pm 5.43$} & \multicolumn{2}{|c|}{$116.5 \pm 5.82$} & \multicolumn{2}{|c|}{$114.98 \pm 5.67$} & \multicolumn{2}{|c|}{$110.77 \pm 5.79$} & 0.002 & 0.2 \\
\hline \multicolumn{11}{|c|}{ Systolic blood pressure [mm Hg] } \\
\hline Mean \pm SD & \multicolumn{2}{|c|}{$132.17 \pm 11.94$} & \multicolumn{2}{|c|}{$133.81 \pm 12.53$} & \multicolumn{2}{|c|}{$131.64 \pm 10.38$} & \multicolumn{2}{|c|}{$131.5 \pm 6.97$} & 0.793 & 0.434 \\
\hline \multicolumn{11}{|c|}{ Diastolic blood pressure [mm Hg] } \\
\hline Mean \pm SD & \multicolumn{2}{|c|}{$85.83 \pm 8.42$} & \multicolumn{2}{|c|}{$87.25 \pm 8.81$} & \multicolumn{2}{|c|}{$84.17 \pm 9.22$} & \multicolumn{2}{|c|}{$82.33 \pm 9.35$} & 0.185 & 0.212 \\
\hline
\end{tabular}

$\mathrm{BMI}$ - body mass index; WC - waist circumference; SD - standard deviation

$P_{1}: P$ value for comparing between control and total cases

$P_{2}: P$ value for comparing between patients with DKD and patients without DKD

Statistically significant at $\mathrm{P} \leq 0.05$

reference gene [15]. In the current study, the relative expression of (ABCC8 and Notch 2) genes is compared to a housekeeping gene which was Glyceraldhyde 3-phosphate dehydrogenase gene. The relative gene expression is usually set to 1 for reference samples because ${ }^{\Delta \Delta} \mathrm{CT}$ is equal to 0 and therefore $2^{\circ}$ is equal to 1 . Accordingly, a value less than 1 is considered down expression while more than 1 is overexpression.

\section{Statistical analysis}

Data were fed to the computer and analyzed using IBM SPSS software package version 20.0. (Armonk, NY: IBM Corp). The Kolmogorov-Smirnov test was used to verify the normality of distribution. Significance of the obtained results was judged at the $5 \%$ level The Student t-test was used for normally distributed quantitative variables, to compare between two studied groups while Mann-Whitney test was used for abnormally distributed quantitative variables. Correlations between quantitative continuous variables parameters were evaluated by multivariate regression analysis with analysis of Pearson's coefficients.

\section{Results}

\section{Demographic data}

This study included 80 patients with T2DM including 21 males and 19 females in the group of patients with T2DM with DKD, 23 males and 17 females in the group of patients with T2DM without DKD and 19 males and 21 females in the control group.

The mean age of the studied subjects with T2DM was $53.93 \pm 6.63$ years and $51.37 \pm 9.29$ years in the control group. There was no statistically significant difference between the mean age of the three groups.

Patients with T2DM had higher BMI and WC compared to control group ( $\mathrm{P}=0.001,0.002$ respectively) while there was no significant difference in BMI or WC between patients with DKD and those without DKD. Among the studied groups there was no significant difference regarding mean values of systolic and diastolic blood pressure. The main characteristics of the studied population are described in Table 1.

\section{Laboratory investigations}

Patients with T2DM had significantly higher levels of total cholesterol, triglycerides, LDL-C and CRP in addition to lower levels of HDL-C and eGFR compared to control group while there was no significant difference between them regarding serum creatinine, AST and ALT activity. On the other hand, patients with DKD had significantly higher levels of FPG, $\mathrm{HbA}_{1 c^{\prime}} A C R$, triglycerides, UACR, CRP and significantly lower eGFR compared to patients without DKD while there was no difference between both groups regarding serum creatinine, LDL-C, HDL-C, TC, ALT and AST activity. Basic laboratory investigations are shown in Table 2. 
Table 2. Basic laboratory parameters of the studied groups

\begin{tabular}{|c|c|c|c|c|c|c|}
\hline & \multicolumn{3}{|c|}{ Cases with T2DM } & \multirow{2}{*}{$\begin{array}{l}\text { Control } \\
\text { Group }\end{array}$} & \multirow[t]{2}{*}{$P_{1}$} & \multirow[t]{2}{*}{$P_{2}$} \\
\hline & Total cases & Patients with DKD & Patients without DKD & & & \\
\hline \multicolumn{7}{|l|}{ ALT [U/L] } \\
\hline Mean $\pm S D$ & $21 \pm 6.9$ & $22 \pm 7.8$ & $20 \pm 5.4$ & $19 \pm 6.2$ & 0.431 & 0.140 \\
\hline \multicolumn{7}{|l|}{ AST [U/L] } \\
\hline Mean \pm SD & $18 \pm 5.8$ & $19 \pm 6.1$ & $17 \pm 4.7$ & $17 \pm 4.9$ & 0.562 & 0.272 \\
\hline \multicolumn{7}{|c|}{ Triglycerides [mg/dL] } \\
\hline Mean \pm SD & $206.83 \pm 66.42$ & $233.17 \pm 76.21$ & $180.47 \pm 41.28$ & $121.20 \pm 13.78$ & $<0.001$ & $<0.001$ \\
\hline \multicolumn{7}{|c|}{ Total cholesterol [mg/dL] } \\
\hline Mean \pm SD & $213.69 \pm 33.24$ & $220.38 \pm 30.77$ & $207.0 \pm 34.63$ & $162.78 \pm 21.51$ & $<0.001$ & 0.110 \\
\hline \multicolumn{7}{|c|}{$\mathrm{HDL}-\mathrm{C}[\mathrm{mg} / \mathrm{dL}]$} \\
\hline Mean \pm SD & $48.72 \pm 9.59$ & $46.55 \pm 8.89$ & $50.90 \pm 9.88$ & $55.78 \pm 7.52$ & $<0.001$ & 0.074 \\
\hline \multicolumn{7}{|c|}{ LDL-C [mg/dL] } \\
\hline Mean \pm SD & $138.80 \pm 23.66$ & $142.73 \pm 24.85$ & $134.88 \pm 22.01$ & $93.03 \pm 15.45$ & $<0.001$ & 0.225 \\
\hline \multicolumn{7}{|c|}{ Serum creatinine } \\
\hline Mean \pm SD & $0.79 \pm 0.10$ & $0.79-0.12$ & $0.80 \pm 0.07$ & $0.7 \pm 0.09$ & 0.068 & 0.451 \\
\hline \multicolumn{7}{|c|}{ eGFR $\left[\mathrm{ml} / \mathrm{min} / 1.73 \mathrm{~m}^{2}\right]$} \\
\hline Mean \pm SD & $96.39 \pm 10.92$ & $93.88 \pm 14.05$ & $98.9 \pm 5.60$ & $116.68 \pm 12.54$ & $<0.001$ & 0.041 \\
\hline \multicolumn{7}{|c|}{ UACR $[\mathrm{mg} / \mathrm{gm} . \mathrm{Cr}]$} \\
\hline Mean \pm SD & $123.99 \pm 197.4$ & $237.31 \pm 229.1$ & $10.68 \pm 7.15$ & $8.35 \pm 4.8$ & $<0.001$ & $<0.001$ \\
\hline \multicolumn{7}{|l|}{$\mathrm{HbA}_{1 \mathrm{c}}(\%)$} \\
\hline Mean \pm SD & $9.84 \pm 2.37$ & $10.83 \pm 2.30$ & $8.85 \pm 2.03$ & $5.16 \pm 0.23$ & $<0.001$ & $<0.001$ \\
\hline \multicolumn{7}{|l|}{ FPG [mg/dL] } \\
\hline Mean \pm SD & $207.0 \pm 92.28$ & $230.3 \pm 78.12$ & $183.6 \pm 100.1$ & $82.16 \pm 8.64$ & $<0.001$ & 0.023 \\
\hline \multicolumn{7}{|l|}{ CRP [mg/L] } \\
\hline Mean \pm SD & $50.69 \pm 25.68$ & $59.30 \pm 22.25$ & $42.08 \pm 26.24$ & $7.15 \pm 2.88$ & $<0.001$ & 0.011 \\
\hline
\end{tabular}

ALT — alanine aminotransferase; AST — aspartate aminotransferase; CRP — C-reactive protein; eGFR - estimated glomerular filtration rate; FPG — fasting plasma glucose; $\mathrm{HbA}_{1 \mathrm{c}}$ - hemoglobin $\mathrm{A}_{1 \mathrm{c}} ; \mathrm{HDL}-\mathrm{C}$ - high-density lipoprotein cholesterol; LDL-C — low-density lipoprotein cholesterol; UACR — urinary albumin-to-creatinine ratio

$\mathrm{P}_{1}$ : $\mathrm{P}$ value for comparing between control and total cases

$P_{2}$ : $P$ value for comparing between patients with DKD and patients without DKD

Statistically significant at $\mathrm{P} \leq 0.05$

\section{Gene expression studies}

As regards $A B C C 8$ gene, $92.5 \%(74 / 80)$ of the cases with T2DM showed overexpression while $7.5 \%$ of cases (6/80) showed downregulated expression indicating higher expression of $\mathrm{ABCC} 8$ among patients with T2DM compared to controls. Also, there was a significant positive correlation between $A B C C 8$ gene expression and $\mathrm{HbA}_{1 \mathrm{c}}$ percentage $(\mathrm{P}=0.006)$ while there was no significant correlation between $A B C C 8$ gene expression and other studied parameters. Also, there was no significant difference in the expression of $A B C C 8$ gene between patients with DKD and patients without DKD $(P=0.931)$.

Notch 2 gene showed upregulated expression in $95 \%$ (76/80) of the cases with T2DM and this high expression was demonstrated in patients with DKD as 97.5\% (39/40) of them showed upregulated expression and also in patients without DKD showed upregulated expression of Notch 2 gene in $92.5 \%$ (37/40) of the cases.

Despite the fact that both groups showed overexpression of Notch 2 gene in most of the cases, the expression of Notch 2 gene was significantly higher in patients with DKD compared to those without DKD $(P=0.017)$. Moreover, Notch 2 gene expression was significantly positively correlated with $\mathrm{UACR}, \mathrm{FPG}$ and $\mathrm{HbA}_{1 \mathrm{c}}$ percentage $(\mathrm{P}=0.004,0.15$ and $<0.001$ respectively) and significantly negatively correlated with eGFR $(P=0.002)$ while it did not show any significant correlation with other studied parameters. Expression of both genes among different groups are shown in Tables 3 and 4 and correlation studies are shown in Table 5.

Multivariate regression analysis showed that Notch 2 gene expression was an independent factor associated with both increased UACR $(P=0.008)$ and reduced eGFR $(P=0.003)$. 
Table 3. Expression of ABCC8 and Notch 2 genes among studied groups

\begin{tabular}{|c|c|c|c|c|c|c|}
\hline & \multicolumn{6}{|c|}{ Cases } \\
\hline & \multicolumn{2}{|c|}{ Total $(n=80)$} & \multicolumn{2}{|c|}{ Patients with DKD $(n=40)$} & \multicolumn{2}{|c|}{ Patients without DKD $(n=40)$} \\
\hline & No. & $\%$ & No. & $\%$ & No. & $\%$ \\
\hline \multicolumn{7}{|l|}{ ABCC8 gene } \\
\hline Down expression & 6 & 7.5 & 2 & 5.0 & 4 & 10.0 \\
\hline Over expression & 74 & 92.5 & 38 & 95.0 & 36 & 90.0 \\
\hline \multicolumn{7}{|l|}{ Notch 2 gene } \\
\hline Down expression & 4 & 5.0 & 1 & 2.5 & 3 & 7.5 \\
\hline Over expression & 76 & 95.0 & 39 & 97.5 & 37 & 92.5 \\
\hline
\end{tabular}

Table 4. Comparison between the two groups according to the expression of $A B C C 8$ and Notch 2 genes

\begin{tabular}{lccc}
\hline & $\begin{array}{c}\text { Patients with } \\
\text { DKD }(\mathbf{n}=40)\end{array}$ & $\begin{array}{c}\text { Patients } \\
\text { without } \\
\text { DKD }(\mathbf{n}=40)\end{array}$ & P \\
\hline ABCC8 gene & & $1.46 \pm 0.74$ & 0.931 \\
Mean \pm SD & $1.58 \pm 0.86$ & $1.25(1.09-1.68)$ & \\
Median (IQR) & $1.30(1.09-1.75)$ & \\
Notch 2 gene & & & \\
Mean \pm SD & $1.83 \pm 1.09$ & $1.34 \pm 0.61$ & 0.017 \\
Median (IQR) & $1.65(1.10-2.15)$ & $1.29(1.06-1.58)$ &
\end{tabular}

$P: P$ value for comparing between the studied groups. Statistically significant at $P \leq 0.05$

\section{Discussion}

T2DM is considered a major health problem worldwide. Several risk factors are known to be associated with increased risk of T2DM, including old age, obesity, increased intake of animal fats, sedentary lifestyle, presence of hypertension, hyperlipidemia and positive family history [16].

DKD is an important microvascular complication of T2DM which is usually characterized by increasing urine albumin excretion accompanied by declining eGFR and it is considered a major cause of ESRD worldwide. ADA recommends to assess urinary albumin excretion and eGFR in all patients with T2DM at least annually for screening of DKD [6].

The last years have witnessed advances in our understanding of the genetic basis of T2DM and identification of multiple genetic variants that are associated with T2DM [17]. However, the role of most of the identified genetic variants in the pathogenesis of T2DM and its complications including DKD is still unclear. Moreover other factors seem to contribute to the pathogenesis of T2DM and DKD including possible unidentified genetic factors, unknown environmental and epigenetic factors.
Table 5. Correlation between the expression of ABCC8 and Notch 2 and different studied parameters

\begin{tabular}{lcccc}
\hline Cases $(\mathbf{n = 8 0})$ & \multicolumn{4}{c}{ Fold change } \\
\cline { 2 - 5 } & \multicolumn{2}{c}{ Notch 2 } & \multicolumn{2}{c}{ Abcc8 } \\
\cline { 2 - 5 } & $\mathbf{r}$ & $\mathbf{P}$ & $\mathbf{r}$ & $\mathbf{P}$ \\
\hline BMI & 0.027 & 0.814 & -0.013 & 0.908 \\
FPG & 0.272 & $0.015^{*}$ & 0.100 & 0.375 \\
HbA $_{1 C}$ & 0.534 & $<0.001$ & 0.308 & 0.006 \\
UACR $_{\text {Serum creatinine }}$ & 0.316 & 0.004 & 0.147 & 0.193 \\
Triglycerides & -0.085 & 0.455 & 0.047 & 0.679 \\
eGFR & 0.002 & 0.986 & -0.217 & 0.053 \\
Total cholesterol & 0.159 & 0.159 & -0.016 & 0.891 \\
HDL-C & 0.062 & 0.582 & -0.114 & 0.315 \\
LDL-C & 0.166 & 0.142 & -0.084 & 0.460 \\
CRP & 0.218 & 0.052 & -0.071 & 0.533
\end{tabular}

$\mathrm{BMI}$ - body mass index; CRP — C-reactive protein; eGFR - estimated glomerular filtration rate; $\mathrm{FPG}$ - fasting plasma glucose; $\mathrm{HbA}_{1 \mathrm{c}}$ - hemoglobin $\mathrm{A}_{1 \mathrm{c}} ; \mathrm{HDL}-\mathrm{C}$ - high-density lipoprotein cholesterol; LDL-C — low-density lipoprotein cholesterol; UACR - urinary albumin-to-creatinine ratio; $r-$ Pearson coefficient, statistically significant at $P \leq 0.05$

$A B C C 8$ gene provides instructions for making the sulfonylurea receptor 1 (SUR1) protein which is one subunit of the ATP-sensitive potassium $\left(\mathrm{K}_{\mathrm{ATP}}\right)$ channel that is found across cell membranes in the beta cells of the pancreas [18]. The current study showed increased expression of $A B C C 8$ gene in patients with T2DM compared to normal subjects. This relation may be explained by the fact that changes in the genes that code for potassium channel subunits are expected to change glucose homeostasis by compromising insulin secretion [19].

The results of previous studies investigating the association between ABCC8 gene and T2DM showed conflicting results with some studies showing no association between $A B C C 8$ gene polymorphism and T2DM and other studies and meta-analyses demonstrated 
significant associations of the $A B C C 8$ gene with the susceptibility of T2DM. Vigi et al. [20] enrolled 210 T2DM and 200 non-diabetic subjects for determining the role of polymorphism of $A B C C 8$ gene in T2DM and the findings showed positive association. Another study by Sokolova et al. [21] included 1384 individuals with T2DM and 414 healthy individuals showed that genetic variation located in the $A B C C 8$ region is associated with the development of T2DM. Another meta-analysis by Qin et al. [22] demonstrated that $A B C C 8$ polymorphisms are associated with risk of T2DM in the global population.

The current study also demonstrated a significant positive correlation between $\mathrm{ABCC} 8$ gene expression and $\mathrm{HbA}_{1 \mathrm{c}}$ and this is the first study, up to our knowledge, to demonstrate this correlation in patients with T2DM.

The results of the present study also showed no significant difference in the expression of $A B C C 8$ gene in patients with DKD compared to those without DKD. There are very few studies that investigated the association between $A B C C 8$ gene expression and chronic complications of T2DM. Stefanski et al. [23] conducted a study on 215 obese patients with T2DM and reported no association between the $A B C C 8$ polymorphism gene and the prevalence of chronic diabetic complications in obese patients with long-term T2DM which is in agreement with the present work.

Regarding Notch 2 gene, the current study showed increased expression of Notch 2 gene in patients with T2DM compared to normal subjects and there was a significant positive correlation between Notch 2 gene expression and $\mathrm{HbA}_{1 \mathrm{c}}$ and $\mathrm{FPG}$ indicating not only a possible role of Notch 2 gene expression in the pathogenesis of T2DM but also a negative impact of Notch 2 expression on glycemic control.

A previous meta-analysis linked certain polymorphisms of Notch 2 gene to the development of T2DM [24]. Also, Kim et al. [25] explained that altered expression of Notch 2 is implicated in beta cell dedifferentiation playing a role in islet beta cell regeneration incompetence by increasing apoptosis.

The results of the present study showed that the expression of Notch 2 gene in patients with DKD was higher than those without DKD. Moreover, multivariate regression analysis showed that Notch 2 gene expression had an independent significant positive relation with $\mathrm{UACR}$ and an independent significant negative relation with eGFR indicating a possible role of Notch 2 signaling in the development of DKD. Notch signaling may be induced in mature podocytes under certain pathologic conditions including exposure to high glucose, VEGF or ischemia leading to increased expression of Jag1 as well as re-expression of low levels of Notch receptors including Notch 2 . In mouse models, it seems clear that Notch signaling in the adult, injured glomerulus is pathogenic and in rodents, blockade of Notch signaling decreased Notch target gene expression in the glomerulus and prevented diabetes-induced glomerulosclerosis and podocyte loss by apoptosis [26]. In agreement with current study, Bonegio and Susztak [27] showed that Notch signaling is usually inactive in adult kidney but their signaling is reactivated during course of diabetic nephropathy and it promotes glomerulosclerosis development via increasing glomerular expression of Notch ligand Jag1. Matoba et al. [28] who demonstrated that inhibiting Notch signaling pathway in DKD attenuated podocyte apoptosis. Another study by Murea et al. [29] which included a total of 86 biopsy samples from subjects with acquired kidney diseases including DKD and reported found that Notch 1 and Notch 2 are expressed on podocytes in proteinuric nephropathies and their level of expression correlates with the amount of proteinuria (across all disease groups) indicating a possible relation between Notch pathway and renal pathologies other than DKD. Sharaf et al. [30] in demonstrated significant positive correlation between Notch 2 expression and albuminuria.

The present work and the previously mentioned studies demonstrate that Notch activation may play a role in development of T2DM and DKD and Notch signaling blockade may provide a therapeutic goal for T2DM and DKD.

\section{Conclusion}

Increased expression of Notch 2 and ABCC8 genes may play a role in the pathogenesis of T2DM and they may contribute to poor control of glycemic state. Moreover, altered expression of Notch 2 may play a role in increased susceptibility to DKD and development of albuminuria in patients with T2DM. These results may indicate a possible role of these genes as diagnostic or therapeutic tools in T2DM or DKD. We recommend performing more studies with larger sample sizes and a wider genetic panel to study the expression of different genes in T2DM and its complications, including ABCC 8 and Notch 2, and the possibility of targeting these genes as a therapeutic tool.

\section{Acknowledgement}

This work was supported by Internal Medicine Department (Diabetes Unit), Faculty of Medicine, Alexandria University, Egypt.

\section{Conflict of interest}

The authors declare that there is no conflict of interest. 


\section{REFERENCES}

1. Magliano DJ, Islam RM, Barr ELM, et al. Trends in incidence of total or type 2 diabetes: systematic review. BMJ. 2019; 366: I5003, doi: 10.1136/bmj.15003, indexed in Pubmed: 31511236.

2. International Diabetes Federation. IDF Diabetes Atlas, $9^{\text {th }}$ edn. Brussels, Belgium:International Diabetes Federation, 2019.

3. American Diabetes Association. Diagnosis and classification of diabetes mellitus. Diabetes Care 2019; 42 (Supplement 1): S13-28. 2019

4. Mambiya $M$, Shang $M$, Wang $Y$, et al. The play of genes and nongenetic factors on type 2 diabetes. Front Public Health. 2019; 7: 349, doi: 10.3389/fpubh.2019.00349, indexed in Pubmed: 31803711.

5. Fowler MJ. Microvascular and Macrovascular Complications of Diabetes. Clinical Diabetes. 2011; 29(3): 116-122, doi: 10.2337/ diaclin.29.3.116.

6. American Diabetes Association. Microvascular Complications and Foot Care. Diabetes Care 2019; 42 (Suppl. 1): S124-38.

7. Chang AR, Grams ME, Ballew SH, et al. CKD Prognosis Consortium (CKD-PC), Chronic Kidney Disease Prognosis Consortium, Chronic Kidney Disease Prognosis Consortium. Associations of kidney disease measures with mortality and end-stage renal disease in individuals with and without diabetes: a meta-analysis. Lancet. 2012; 380(9854): 1662-1673, doi: 10.1016/S0140-6736(12)61350-6, indexed in Pubmed: 23013602.

8. Busiah $\mathrm{K}$, Verkarre V, Cavé $\mathrm{H}$, et al. Human pancreas endocrine cell populations and activating ABCC8 mutations. Horm Res Paediatr. 2014; 82(1): 59-64, doi: 10.1159/000360004, indexed in Pubmed: 24941889.

9. Tinker A, Aziz Q, Li Y, et al. ATP-sensitive potassium channels and their physiological and pathophysiological roles. Compr Physiol. 2018; 8(4): 1463-1511, doi: 10.1002/cphy.c170048, indexed in Pubmed: 30215858.

10. Bolós V, Grego-Bessa J, de la Pompa JL. Notch signaling in development and cancer. Endocr Rev. 2007; 28(3): 339-363, doi: 10.1210/er.2006-0046, indexed in Pubmed: 17409286.

11. Siebel $C$, Lendahl U. notch signaling in development, tissue homeostasis, and disease. Physiol Rev. 2017; 97(4): 1235-1294, doi: 10.1152/physrev.00005.2017, indexed in Pubmed: 28794168.

12. Florez JC. Leveraging genetics to advance type 2 diabetes prevention. PLoS Med. 2016; 13(7): e1002102, doi: 10.1371/journal. pmed.1002102, indexed in Pubmed: 27458973.

13. Florez JC. Mining the genome for therapeutic targets. Diabetes. 2017; 66(7): 1770-1778, doi: 10.2337/dbi16-0069, indexed in Pubmed: 28603140.

14. Levey AS, Stevens LA. Estimating GFR using the CKD Epidemiology Collaboration (CKD-EPI) creatinine equation: more accurate GFR estimates, lower CKD prevalence estimates, and better risk predictions. Am J Kidney Dis. 2010; 55(4): 622-627, doi: 10.1053/j. ajkd.2010.02.337, indexed in Pubmed: 20338463.

15. Kuang J, Yan Xu, Genders AJ, et al. An overview of technical considerations when using quantitative real-time PCR analysis of gene expression in human exercise research. PLoS One. 2018; 13(5): e0196438, doi: 10.1371/journal.pone.0196438, indexed in Pubmed: 29746477.

16. DeFronzo RA, Ferrannini E, Groop L, et al. Type 2 diabetes mellitus. Nat Rev Dis Primers. 2015; 1: 15019, doi: 10.1038/nrdp.2015.19, indexed in Pubmed: 27189025.
17. Scott RA, Langenberg C, Sharp SJ. The link between family history and risk of type 2 diabetes is not explained by anthropometric, lifestyle or genetic risk factors: the EPIC-InterAct study. Diabetologia. 2013; 56 (1): 60-69, doi: 10.1007/s00125-012-2715-x, indexed in Pubmed: 22526603.

18. Vana DR, Adapa D, Prasad VS, et al. Diabetes mellitus types: key genetic determinants and risk assessment. Genetics and Molecular Research. 2019; 18(2), doi: 10.4238/gmr16039952.

19. Bonfanti DH, Alcazar LP, Arakaki PA, et al. ATP-dependent potassium channels and type 2 diabetes mellitus. Clin Biochem. 2015; 48(7-8): 476-482, doi: 10.1016/j.clinbiochem.2014.12.026, indexed in Pubmed: 25583094.

20. Viji D, Aswathi P, Charmine PP, et al. Genetic association of ABCC8 rs757110 polymorphism with Type 2 Diabetes Mellitus risk: A case-control study in South India and a meta-analysis. Gene Reports. 2018; 13: 220-228, doi: 10.1016/j.genrep.2018.10.015.

21. Sokolova EA, Bondar IA, Shabelnikova OY, et al. Replication of KCNJ11 (p.E23K) and ABCC8 (p.S1369A) Association in Russian Diabetes Mellitus 2 Type Cohort and Meta-Analysis. PLoS One. 2015; 10(5): e0124662, doi: 10.1371/journal.pone.0124662, indexed in Pubmed: 25955821.

22. Qin $\amalg$, Lv Y, Huang QY. Meta-analysis of association of common variants in the KCNJ11-ABCC8 region with type 2 diabetes. Genet Mol Res. 2013; 12(3): 2990-3002, doi: 10.4238/2013. August.20.1, indexed in Pubmed: 24065655.

23. Stefanski A, Majkowska L, Ciechanowicz A, et al. The common C49620T polymorphism in the sulfonylurea receptor gene $(A B C C 8)$, pancreatic beta cell function and long-term diabetic complications in obese patients with long-lasting type 2 diabetes mellitus. Exp Clin Endocrinol Diabetes. 2007; 115(5): 317-321, doi: 10.1055/s-2007-967086, indexed in Pubmed: 17516295.

24. Zeggini E, Scott $U$, Saxena R, et al. Wellcome Trust Case Control Consortium. Meta-analysis of genome-wide association data and large-scale replication identifies additional susceptibility loci for type 2 diabetes. Nat Genet. 2008; 40(5): 638-645, doi: 10.1038/ ng.120, indexed in Pubmed: 18372903.

25. Kim W, Shin YK, Kim BJ, et al. Notch signaling in pancreatic endocrine cell and diabetes. Biochem Biophys Res Commun. 2010; 392(3): 247-251, doi: 10.1016/j.bbrc.2009.12.115, indexed in Pubmed: 20035712.

26. Niranjan T, Bielesz B, Gruenwald A, et al. The Notch pathway in podocytes plays a role in the development of glomerular disease. Nat Med. 2008; 14(3): 290-298, doi: 10.1038/nm1731, indexed in Pubmed: 18311147

27. Bonegio R, Susztak K. Notch signaling in diabetic nephropathy. Exp Cell Res. 2012; 318(9): 986-992, doi: 10.1016/j. yexcr.2012.02.036, indexed in Pubmed: 22414874.

28. Matoba K, Kawanami D, Nagai Y, et al. Rho-kinase blockade attenuates podocyte apoptosis by inhibiting the notch signaling pathway in diabetic nephropathy. Int J Mol Sci. 2017; 18(8), doi: 10.3390/ijms18081795, indexed in Pubmed: 28820432.

29. Murea M, Park JK, Sharma S, et al. Expression of Notch pathway proteins correlates with albuminuria, glomerulosclerosis, and renal function. Kidney Int. 2010; 78(5): 514-522, doi: 10.1038/ ki.2010.172, indexed in Pubmed: 20531454.

30. Sharaf SA, Kantoush NA, Ayoub DF, et al. Altered expression of WFS1 and NOTCH2 genes associated with diabetic nephropathy in T2DM patients. Diabetes Res Clin Pract. 2018; 140: 304-313, doi: 10.1016/j.diabres.2018.03.053, indexed in Pubmed: 29626590. 